\title{
Persistent Epidural Fluid at the Cervical Spine Level, Lessons from Radiology
}

\author{
Madhumani N. Rupasingh, Naveen Vanga, Aseem R. Hemmad, Sonya A. Johnson \\ Department of Anesthesiology, University of Texas Health Science Centre, Houston, USA \\ Email: Madhumani.Rupasinghe@uth.tmc.edu
}

Received 5 June 2015; accepted 22 June 2015; published 29 June 2015

Copyright (C) 2015 by authors and OALib.

This work is licensed under the Creative Commons Attribution International License (CC BY). http://creativecommons.org/licenses/by/4.0/

(C) (i) Open Access

\begin{abstract}
Following an accidental dural puncture during the placement of epidural anesthesia, leakage of cerebrospinal fluid occurs. This leads to tugging on intracranial pain-sensitive structures and causes a headache that is postural in nature. According to radiology literature, the presence of retro spinal fluid collections has been associated with the diagnosis of spontaneous intracranial hypotension. The characteristic C1-C2 radiographic sign, called the C1-C2 false localizing sign, can be found on magnetic resonance (MR) images as a focal area of fluid-like signal intensity and on CT myelograms as a CSF collection between the spinous processes of $\mathrm{C} 1$ and C2. As our case report demonstrates, this sign is also associated with intracranial hypotension after lumbar puncture.
\end{abstract}

\section{Keywords}

Post Dural Puncture Headache, MRI Imaging

Subject Areas: Anaesthesiology \& Pain Management

\section{Introduction}

The pathophysiology of post dural puncture headache (PDPH) is unclear. It is thought to develop as a result of leakage of cerebrospinal fluid (CSF) through a hole in the dura. After the dura is accidentally punctured during placement of an epidural catheter, CSF leakage occurs and leads to tugging on intracranial pain-sensitive structures. This causes a bilateral, fronto-temporal, retro-orbital or occipital headache that can extend into the neck. It is associated with nausea, vomiting and photophobia. The distinguishing sign is the postural component of the symptoms. It is aggravated by sitting or standing and relieved by lying flat. It can begin up to 72 hours after the initial dural injury and can last for weeks. When conservative measures fail, epidural blood patch may be required. Magnetic resonance imaging (MRI) of the brain post dural puncture normally demonstrate dural enhancement with low lying cerebellar tonsils, and venous distention. The spinal MRI findings have been described as spinal meningeal enhancement and dilated epidural veins [1]. According to radiology literature, the 
presence of retro-spinal fluid collections, thought to be a transudate, can also help to establish the diagnosis of intracranial hypotension.

\section{Case Description}

A healthy 28-year-old Caucasian parturient with a BMI of 42 was admitted to the labor and delivery floor, requesting an epidural for pain management. The epidural space was found after three attempts, using loss of resistance to saline. Following a negative test dose, the epidural infusion was initiated and the patient received satisfactory labor analgesia. The patient subsequently delivered uneventfully and was transferred to the post partum unit.

Twelve hours post delivery the patient began to complain of headache and neck pain, described as 6/10 in intensity, and only mildly increased in the upright position. The neck pain improved with bed rest, hydration and analgesics. However, approximately twenty four hours later, the patient reported an acute worsening of her neck pain when she attempted ambulation to the shower. Due to the somewhat unusual presentation of symptoms and elevated patient concern, an MRI of the brain and neck was obtained (Figure 1).

Magnetic resonance imaging in this case showed the presence of a dark epidural collection (indicated by green arrows), possibly fluid, partially collapsing the thecal sac from C2-3 through the lowest level of the included cervical spine, with some persistent fluid surrounding the cord. This was thought to indicate dilated epidural veins relating to spontaneous intracranial hypotension or alternatively, epidural fluid.

After the results of the MRI, an epidural blood patch was performed successfully and uneventfully. The patient expressed improvement in symptoms and was later discharged home.

\section{Discussion}

CSF leakage distributed around nerve roots and paraspinal areas at the lumbosacral level is a relatively common finding in patients after a dural puncture. These fluid collections are the result of CSF escaping from the subarachnoid space into the paraspinal loose connective tissues. The C1-C2 radiographic sign, also called the C1-

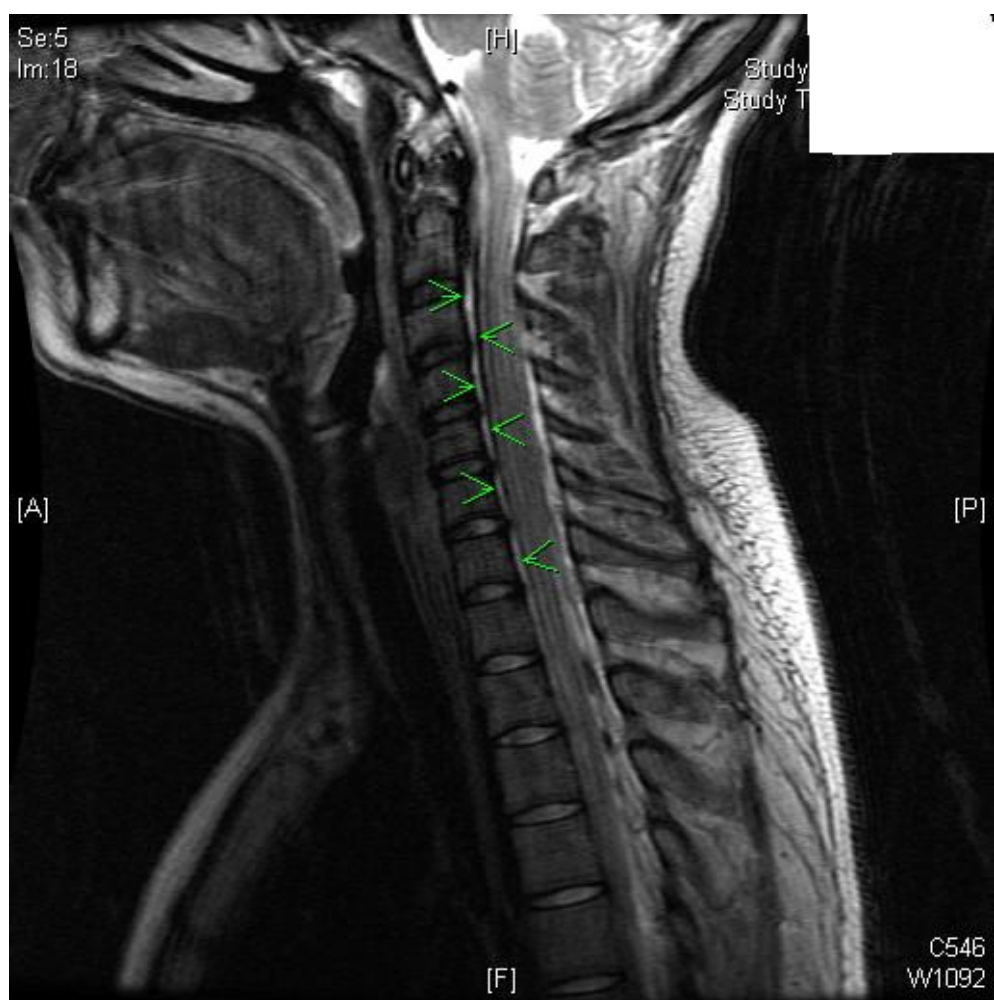

Figure 1. Dark epidural collection, indicated by green arrows. (IMAGE AS SEPARATE FILE). 
C2 false localizing sign, is reported on MR images as a focal area of fluid-like signal intensity and on CT myelograms as a CSF collection between the spinous processes of C1 and C2. Radiology literature describes the visualization of this finding leading to the diagnosis of spontaneous intracranial hypotension. This sign has also been reported in association with intracranial hypotension after lumbar puncture. Hence it leads to the assertion that this retrospinal fluid in the cervical spine does not represent direct CSF leakage and may be secondary to the lumbar puncture itself.

Two theories explain the origin of this retro-spinal fluid collection at C1-C2. First, the fluid collection is thought to be due to CSF leakage. Second, it is thought to be a transudate resulting from hydrostatic pressure changes. There is a twofold mechanism for the transudate formation. In order to maintain a constant intraspinal volume to compensate for the decrease in CSF volume, in accordance with the Monro-Kellie assumption, the venous plexus dilate, mainly at the C1-C2 level. If the dilatation of the venous plexus is restricted, or not sufficient, transudate is thought to collect subdurally.

The suboccipital C1-C2 region is most prone to the formation of a substantial transudate due to a number of reasons. The high density of venous structures, their proximity and functional connectivity with the cranial sinuses, lack of epidural fat, laxity of the connective tissues at this level and the mobility of the segment due to lack of osseous support seems to make this region more prone to the escape of fluid [2] [3].

\section{Conclusion}

CSF hypovolemia following a dural leak commonly manifests with postural headache. Without a documented dural puncture and unusual presentation of symptoms, treatment can be guided by investigations and imaging. Although most radiologically visualized CSF leakages are not associated with PDPH and do not require treatment, it is of importance to understand and appreciate this presentation to avoid misdiagnosis. As in our case, knowledge of these MRI findings will aid Anesthesiologists in the early diagnosis of CSF hypovolemia, and facilitate the institution of relevant therapy in the absence of more definitive clinical findings.

(Patients informed consent was obtained for the publication of this case report.)

\section{References}

[1] Yousry, I., Förderreuther, S., Moriggl, B., Holtmannspötter, M., Naidich, T.P., Straube, A. and Yousry, T.A. (2001) Cervical MR Imaging in Postural Headache: MR Signs and Pathophysiological Implications. AJNR American Journal of Neuroradiology, 22, 1239-1250.

[2] Vadi, S. and Kumar, D. (2009) Pathophysiological Basis of Radiological Findings in Cerebrospinal Fluid Hypovolemia-A Case Report and Review of the Literature. The Internet Journal of Internal Medicine, 8, Number 1.

[3] Medina, J.H., Abrams, K., Falcone, S. and Bhatia, R.G. (2010) Spinal Imaging Findings in Spontaneous Intracranial Hypotension. AJNR American Journal of Neuroradiology, 195, 459-464. http://dx.doi.org/10.2214/AJR.09.3289 